Research Article

\title{
Investigation on a Duct Noise Control Method through Membranes in Tandem
}

\author{
Cheng Shen, ${ }^{1,2,3}$ Shasha Yang $\mathbb{D}^{3,4}$ Harris K. H. Fan, ${ }^{5}$ and Randolph C. K. Leung $\mathbb{D}^{5}$ \\ ${ }^{1}$ College of Aerospace Engineering, Nanjing University of Aeronautics and Astronautics, Nanjing 210016, China \\ ${ }^{2}$ Space Structure and Mechanism Technology Laboratory of China Aerospace Science and Technology Group Co., Ltd., \\ Shang Hai, China \\ ${ }^{3}$ State Key Laboratory for Strength and Vibration of Mechanical Structures, Xi'an Jiaotong University, Xi'an 710049, China \\ ${ }^{4}$ School of Mechanical Engineering, Nanjing Vocational University of Industry Technology, Nanjing 210023, China \\ ${ }^{5}$ Department of Mechanical Engineering, The Hong Kong Polytechnic University, Hung Hom, Kowloon, Hong Kong, China
}

Correspondence should be addressed to Shasha Yang; 2016100849@niit.edu.cn and Randolph C. K. Leung; mmrleung@ polyu.edu.hk

Received 1 March 2021; Revised 19 March 2021; Accepted 25 June 2021; Published 16 July 2021

Academic Editor: Roberto Nascimbene

Copyright (C) 2021 Cheng Shen et al. This is an open access article distributed under the Creative Commons Attribution License, which permits unrestricted use, distribution, and reproduction in any medium, provided the original work is properly cited.

Duct noise control is an important practical problem. This paper explores the vibro-acoustic mechanism of duct noise through the membrane in tandem. Validity and feasibility of the proposed analytical model is demonstrated by comparing existing simplified models and full direct aeroacoustic simulation solved with the CE/SE model. It is shown that the coupling effect between two membranes in tandem is not negligible to predict system response. Moreover, introduction of multimembranes is important or even the only efficient way to apply this passive control method in practice.

\section{Introduction}

How to obtain better duct noise control method has long been a long-term studying problem in industrial field [1-3]. It is known that two traditional passive control methods (i.e., expansion-chamber design and duct lining) have their own disadvantage. Specifically, there exist periodic pass bands for the expansion-chamber case, while the duct lining method is not efficient in the low frequency range. In 2001, Huang [4] creatively proposed a new passive control method theoretically based on wave reflection and structural damping of a flexible membrane inspired by the investigation on flexible tube in human respiratory systems. Furthermore, a series of systematic research studies have done by Huang et al., such as investigation on detailed physical mechanism of flexible membrane [5], drum-like design to avoid break-out noise [6], and practical experimental test work. Besides, other researchers also conduct many valuable works recently. $\mathrm{Du}$ et al. [8] have discussed the influence of different boundary constraints on sound transmission loss by a modified improved Fourier series method. Yu et al. [9] replaced ordinary membrane with an electro-active membrane (i.e., dielectric elastomer) and extended this passive control method into adaptive-passive control strategy. Recently, Fan et al. [10-12] conducted systematic numerical analysis on Huang's flexible membrane model from the viewpoint of aeroacousticstructural interaction based on the conservation element and solution element (CE/SE) method; they found that the presence of flow with the incident wave in the flow direction mainly weakens the reflection.

For the practical application of flexible membrane, the request of high tension force may be one of major barriers since larger tension force can produce better sound insulation effect [4]. To solve this problem, our idea is to introduce another membrane in tandem. Both an analytical model and CE/SE method are built to explore the role of additional membrane in consideration of complete coupling effect between two membranes. In detail, the frequency domain model and time domain model are described in Section 2 and Section 3, respectively. Validation of the proposed model and corresponding discussion are given in Section 4. Conclusions are summarized in Section 5. 


\section{Frequency Domain Model}

As shown in Figure 1, the first membrane is assumed to be hinged at $x= \pm L_{1} / 2$, where $L_{1}=\widehat{L}_{1} / \widehat{h}_{0}$ is the dimensionless membrane length. An incident plane sound wave is assumed coming from left to right with unit amplitude:

$$
P_{i}=e^{i\left(\omega t-k_{0} t\right)} .
$$

Governing dynamic equations of the membranes 1 and 2 are written as

$$
\left\{\begin{array}{l}
M_{1} \frac{\partial^{2} \eta_{1}}{\partial t^{2}}+D_{1} \frac{\partial \eta_{1}}{\partial t}+P_{i}+Z_{11} \frac{\partial \eta_{1}}{\partial t}+Z_{21} \frac{\partial \eta_{2}}{\partial t}+T_{1} \frac{\partial^{2} \eta_{1}}{\partial x^{2}}=0 \\
M_{2} \frac{\partial^{2} \eta_{2}}{\partial t^{2}}+D_{2} \frac{\partial \eta_{2}}{\partial t}+P_{i}+Z_{22} \frac{\partial \eta_{2}}{\partial t}+Z_{12} \frac{\partial \eta_{2}}{\partial t}+T_{2} \frac{\partial^{2} \eta_{2}}{\partial x^{2}}=0
\end{array}\right.
$$

where $\eta_{i}, M_{i}, D_{i}$, and $T_{i}(i=1,2)$ are, respectively, displacement, mass ratio, dimensionless damping coefficient, and tension force. $Z_{11}$ and $Z_{22}$ are local radiation impedance structure itself. $Z_{21}$ and $Z_{12}$ are mutual radiation impedance which reflect the coupling effect in this kind of the structural acoustic system. The standard Galerkin method is employed to solve equations above. If velocity $V_{i}=\partial \eta_{i} / \partial t$ is defined, then $V_{i}$ can be expanded in terms of sine transform using two local dimensionless variable $\xi_{1}=x / L_{1}+1 / 2$, and $\xi_{2}=x / L_{2}-L_{1} / 2 L_{2}-L_{m} / L_{2}$; then, both $\xi_{1}$ and $\xi_{2}$ span from 0 to 1 for membrane 1 and membrane 2 :

$$
\begin{aligned}
V_{1} & =\sum_{j=1}^{\infty} V_{1 j} \sin j \pi \xi_{1}, \\
V_{1 j} & =2 \int_{0}^{1} V_{1}\left(\xi_{1}\right) \sin j \pi \xi_{1} \mathrm{~d} \xi_{1}, \\
V_{2} & =\sum_{k=1}^{\infty} V_{2 k} \sin j \pi \xi_{2}, \\
V_{2 k} & =2 \int_{0}^{1} V_{2}\left(\xi_{2}\right) \sin k \pi \xi_{2} \mathrm{~d} \xi_{2} .
\end{aligned}
$$

After sine transform, equations (2) can be reduced to a set of linear equations:

$$
\begin{cases}L L_{1 j} V_{1 j}+\sum_{n_{1}=1}^{N_{1}} V_{1 n_{1}} Z_{11}\left(j, n_{1}\right)+\sum_{n_{2}=1}^{N_{2}} V_{2 n_{2}} Z_{21}\left(j, n_{2}\right)=-I_{j}, & j=1,2,3 \cdots N_{1}, \\ L L_{2 k} V_{2 k}+\sum_{n_{1}=1}^{N_{1}} V_{1 n_{1}} Z_{12}\left(k, n_{1}\right)+\sum_{n_{2}=1}^{N_{2}} V_{2 n_{2}} Z_{22}\left(k, n_{2}\right)=-I_{k}, & k=1,2,3 \cdots N_{2},\end{cases}
$$

where $\quad L L_{1 j}=i M_{1} \omega+D_{1}+M_{1} c_{1 T}^{2} / i \omega\left(j \pi / L_{1}\right)^{2}$, $L L_{2 k}=i M_{2} \omega+D_{2}+M_{2} c_{2 T}^{2} / i \omega\left(k \pi / L_{2}\right)^{2}, c_{1 T}$ and $c_{2 T}$ are wave speed of these two membranes, respectively, and $I_{j}$ is the sine ${ }_{1}^{1}$ transform of incident ${ }_{1}^{1}$ wave, i.e., $I_{j}=2 \int_{0}^{1} P_{i} \sin \left(j \pi \xi_{1}\right) d \xi_{1}$ and $I_{k}=2 \int_{0}^{1} P_{i} \sin \left(k \pi \xi_{2}\right) d \xi_{2}$. Detailed expressions of local radiation impedance can be found in Huang's paper and not shown here for brevity.
And, mutual modal radiation impedance will be given in the following.

2.1. Mutual Modal Radiation Impedance. The starting point is the calculation of radiated sound pressure by vibrating membrane. Formula [4] is used as follows:

$$
p(x, y, t)=\frac{1}{2} \sum_{m=0}^{\infty} c_{m} \psi_{m}(y) \int_{-L / 2}^{L / 2} \psi_{m}\left(y^{\prime}\right) V\left(x^{\prime}, t\right) \times\left[H\left(x-x^{\prime}\right) e^{\left(-i \omega\left(x-x^{\prime}\right) / c_{m}\right)}+H\left(x^{\prime}-x\right) e^{\left(i \omega\left(x-x^{\prime}\right) / c_{m}\right)}\right] d x^{\prime}
$$

where $H$ is the Heaviside function, $(x, y)$ and $\left(x^{\prime}, y^{\prime}\right)$ are coordinates of exciting point and response point, $\psi_{m}(y)=$ $\sqrt{2-\delta_{0 m}} \cos (m \pi y)$ is the channel modal function, $\delta$ is the
Kronecker delta, and $c_{m}=i / \sqrt{(m \pi / \omega)^{2}-1}$ is the complex modal wave speed. Then, mutual modal radiation impedance $Z_{21}$ and $Z_{12}$ can be expressed as 


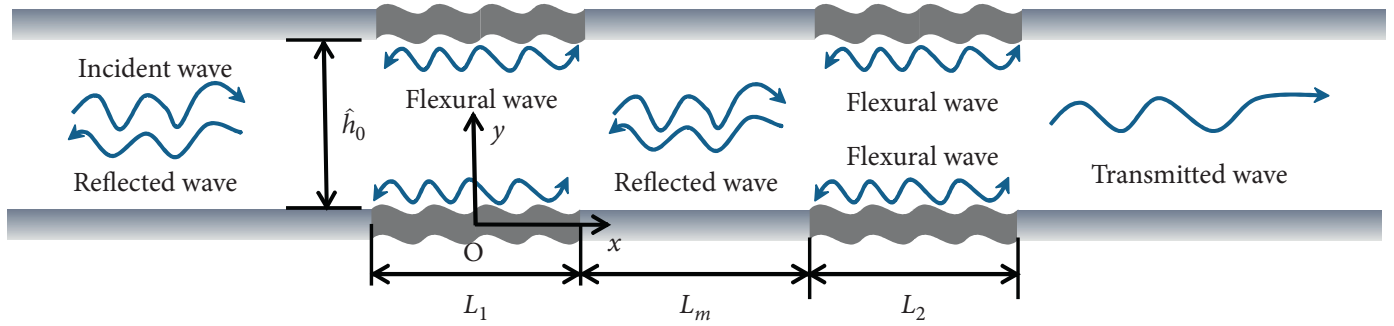

FIgURE 1: Illustration of duct noise control setup through the membranes in tandem.

$$
\begin{aligned}
& Z_{21}\left(j, n_{2}\right)=2 \int_{0}^{1} p_{n_{2}} \sin \left(j \pi \xi_{2}\right) d \xi_{2}=L_{2} \sum_{m_{2}=0}^{\infty} c_{m_{2}}\left(2-\delta_{0 m_{2}}\right) \\
& \times \int_{0}^{1} \sin \left(n_{2} \pi \xi_{2}^{\prime}\right)\left[H\left(x_{1}-x_{2}^{\prime}\right) e^{\left(-i \omega\left(x_{1}-x_{2}^{\prime}\right) / c_{m_{2}}\right)}+H\left(x_{2}^{\prime}-x_{1}\right) e^{\left(i \omega\left(x_{1}-x_{2}^{\prime}\right) / c_{m_{2}}\right)}\right] d \xi_{2}^{\prime} \int_{0}^{1} \sin \left(j \pi \xi_{1}\right) d \xi_{1}, \\
& Z_{12}\left(k, n_{1}\right)=2 \int_{0}^{1} p_{n_{1}} \sin \left(k \pi \xi_{1}\right) d \xi_{1}=L_{1} \sum_{m_{1}=0}^{\infty} c_{m_{1}}\left(2-\delta_{0 m_{1}}\right) \\
& \left.\times \int_{0}^{1} \sin \left(n_{1} \pi \xi_{1}^{\prime}\right)\left[H\left(x_{2}-x_{1}^{\prime}\right) e^{\left(-i \omega\left(x_{2}-x_{1}^{\prime}\right) / c_{m_{1}}\right)}+H\left(x_{1}^{\prime}-x_{2}\right) e^{\left(i \omega\left(x_{2}-x_{1}^{\prime}\right) / c_{m_{1}}\right.}\right)\right] d \xi_{1}^{\prime} \int_{0}^{1} \sin \left(k \pi \xi_{2}\right) d \xi_{2},
\end{aligned}
$$

where channel modal function $\psi_{m}(y)$ has degenerated to $\sqrt{2-\delta_{0 m}}$ since exciting and response point located at $y=0$ and $y^{\prime}=0$. It is noted that $x_{2}^{\prime}>x_{1}$ and $x_{2}>x_{1}^{\prime}$ always exist once the present coordinate system is built; then, the Heaviside function can be easy to calculate. The rest of the derivation process is routine and not be repeated here.

2.2. Sound Transmission Loss. Once the linear equation (4) are solved, according to equation (5), the expanded velocity terms can be used to predict radiated sound pressure $p_{\text {rad }}$, which consists of two parts from membrane 1 and membrane 2; then, the transmission loss is defined as

$$
\mathrm{STL}=-20 \log 10\left|\frac{p_{i}+p_{\mathrm{rad}}}{p_{i}}\right| .
$$

\section{Time Domain Model}

An aeroacoustic model based on direct aeroacoustic simulation (DAS) approach is also employed in the present study. The aeroacoustic problem is governed by the twodimensional compressible Navier-Stokes equations together with ideal gas law for calorically perfect gas. The normalized Navier-Stokes equations without the source can be written in the strong conservation form as follows:

$$
\frac{\partial \mathbf{U}}{\partial t}+\frac{\partial\left(\mathbf{F}-\mathbf{F}_{v}\right)}{\partial x}+\frac{\partial\left(\mathbf{G}-\mathbf{G}_{v}\right)}{\partial y}=0
$$

where $\quad \mathbf{U}=\left[\begin{array}{llll}\rho & \rho u & \rho v & \rho E\end{array}\right]^{T}, \quad \mathbf{F}=\left[\begin{array}{ll}\rho u & \rho u^{2}+p \rho u v\end{array}\right.$ $(\rho E+p) u]^{T}, \quad \mathbf{G}=\left[\begin{array}{llll}\rho v & \rho u v & \rho v^{2}+p & (\rho E+p) v\end{array}\right]^{T}, \quad \mathbf{F}_{v}=$ $1 / \operatorname{Re}\left[\begin{array}{llll}0 & \tau_{x x} & \tau_{x y} & \tau_{x x} u+\tau_{x y} v-q_{x}\end{array}\right]^{T}$, and

$$
\mathbf{G}_{v}=\frac{1}{\operatorname{Re}}\left[\begin{array}{llll}
0 & \tau_{x y} & \tau_{y y} & \tau_{x y} u+\tau_{y y} v-q_{y}
\end{array}\right]^{T} .
$$

$\rho$ is the density of fluid, $u$ and $v$ are the velocities in $x$ and $y$ direction, respectively, $t$ is the time, stress $\tau_{x x}=(2 / 3) \mu(2 \partial u / \partial x-\partial v / \partial y), \quad \tau_{x y}=\mu(2 \partial u / \partial y-\partial v / \partial x)$, and $\quad \tau_{y y}=(2 / 3) \mu(2 \partial v / \partial y-\partial u / \partial x)$, total energy $E=p / \rho(\gamma-1)+\left(u^{2}+v^{2}\right) / 2$, pressure $p=\rho T / \gamma M^{2}$, heat flux $\quad q_{x}=\left[\mu /(\gamma-1) \operatorname{Pr} M^{2}\right](\partial T / \partial x), \quad q_{y}=[\mu /(\gamma-1)$ $\left.\operatorname{Pr} M^{2}\right](\partial T / \partial y)$, the specific heat ratio $\gamma=1.4$, Mach number $M=\widehat{u}_{0} / \widehat{c}_{0}$, where $\widehat{u}_{0}$ is the duct mean flow velocity, and $\widehat{c}_{0}=\sqrt{\gamma \widehat{R} \widehat{T}_{0}}$, specific gas constant for $\widehat{R}=287.058 \mathrm{~J} /(\mathrm{kg} \cdot \mathrm{K})$. Choosing a reference length $\widehat{L}_{0}$, a reference density $\widehat{\rho}_{0}$, a reference acoustic speed $\hat{c}_{0}$, Reynolds number $\operatorname{Re}=\widehat{\rho}_{0} \widehat{c}_{0} \widehat{L}_{0} / \widehat{\mu}_{0}$, and Prandtl number $\operatorname{Pr}=\widehat{c}_{p, 0} \widehat{\mu}_{0} / \widehat{k}_{0}=0.71$, the Navier-Stokes equations are solved by the CE/SE-based DAS solver. Detailed implementation progress can be found in Fan and Leung's another work [10].

\section{Numerical Results and Discussion}

4.1. Validation of Model. Firstly, Huang's model [4] is employed to validate present vibro-acoustic model preliminary. In the following discussions, the same parameters are chosen as the default. The height of duct $\widehat{h}_{0}=0.1 \mathrm{~m}$, mass per unit length of membrane $\widehat{M}=0.05 \mathrm{~kg} / \mathrm{m}^{2}$, tension force $\widehat{T}=58.06 \mathrm{~N} / \mathrm{m}$, the length of membrane 1 and 2 , and space is $L_{1}=L_{2}=L_{m}=5$. Since Huang's analytical model refers to one membrane setup, the second membrane is neglected in real calculation to approximate Huang's result. As shown in Figure 2, good agreement between the present degenerative model and Huang model is achieved, which validate present frequency domain model primarily.

The significant STL reduction at frequency $f=0.36$ is attributed to the critical frequency phenomenon where wavenumber of the flexural wave is equal to acoustic wavenumber of surrounding fluid. If frequency is higher than critical frequency, supersonic radiation sound wave 


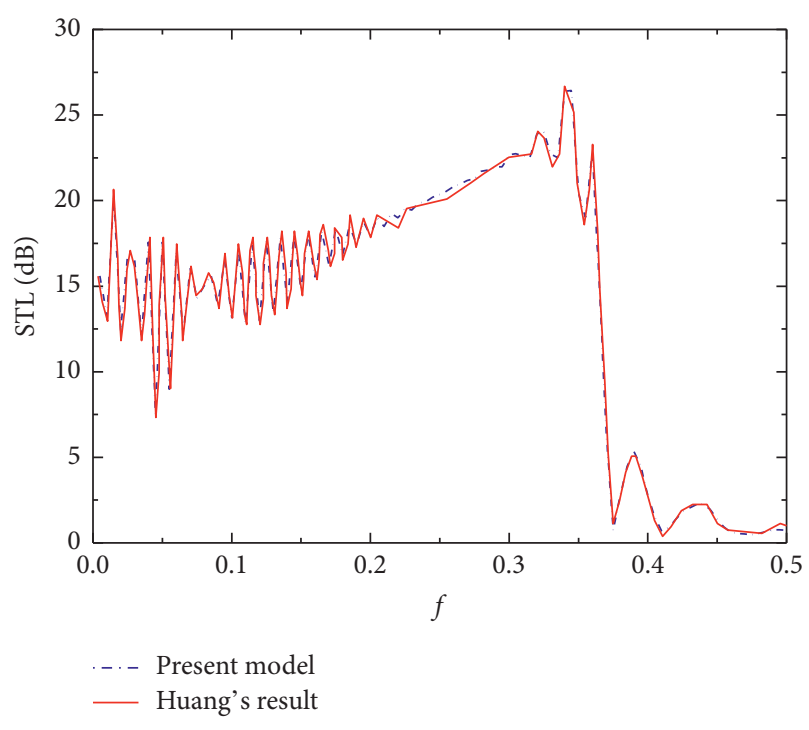

FIgURE 2: Sound transmission loss of one membrane setup.

cannot provide effective wave reflection; then, most of sound wave energy will propagate to downstream except consumed part because of structural damping.

4.2. Frequency Domain Model vs. Time Domain Model. Furthermore, the present frequency domain model is compared to the time domain method to further illustrate the validity of the model. Here, two acoustic response point at $x=5$ and $x=20$ are chosen to reflect the sound insulation effect of these two membranes, as shown in Figures 3 and 4, respectively. Generally, it is shown that excellent agreement between present two models can be achieved including oscillations' peaks and troughs on the curves, which validate the present analytical model and the numerical model effectively again. The quantitative difference between them is mainly attributed to the structural damping model of the membrane. Specifically, the modal damping model in the frequency domain model cannot be realized completely in the time domain model. More detailed explanation can be found in [10-12].

\subsection{Frequency Domain Model vs. Transfer Matrix Method.} For the multimembrane cases, another straightforward way is to use the transfer matrix method, which neglects the coupling effect between different membranes, as shown in Choy and Huang's work [13]. Therefore, it is significant to discuss the coupling effect of membranes in tandem through the comparison between the transfer matrix method and the present coupled model. Intuitively, the coupling effect varies with the distance of two membranes. As shown in Figure 5, the discrepancy between two models decrease with increasing spacing when incident dimensionless frequency $f=0.2$, and the maximum gap (about $4 \mathrm{~dB}$ ) happens when the gap is zero, i.e., the membranes are arranged continuously. By the way, the coupling effect for the whole system is embodied in the mutual modal radiation impedance terms $Z_{12}$ and $Z_{21}$ from the mathematical point of view.

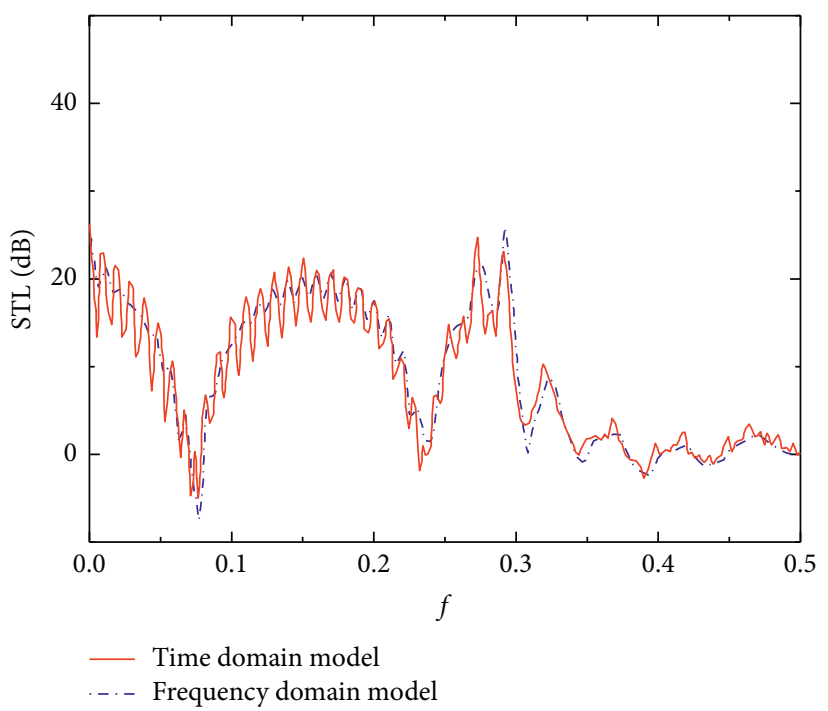

FIGURE 3: Comparison of STL for radiated sound pressure at $x=5$.

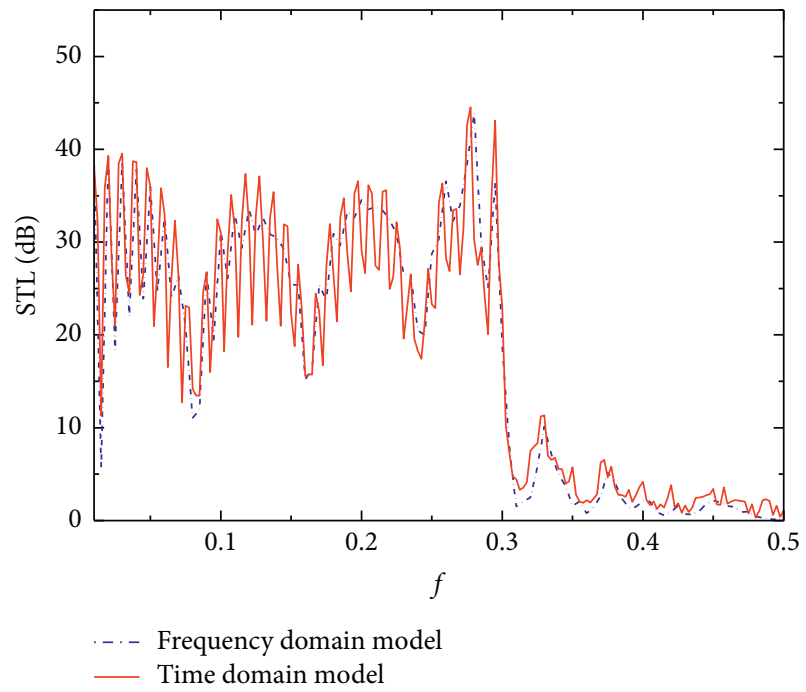

FIGURE 4: Comparison of STL for radiated sound pressure at $x=20$.

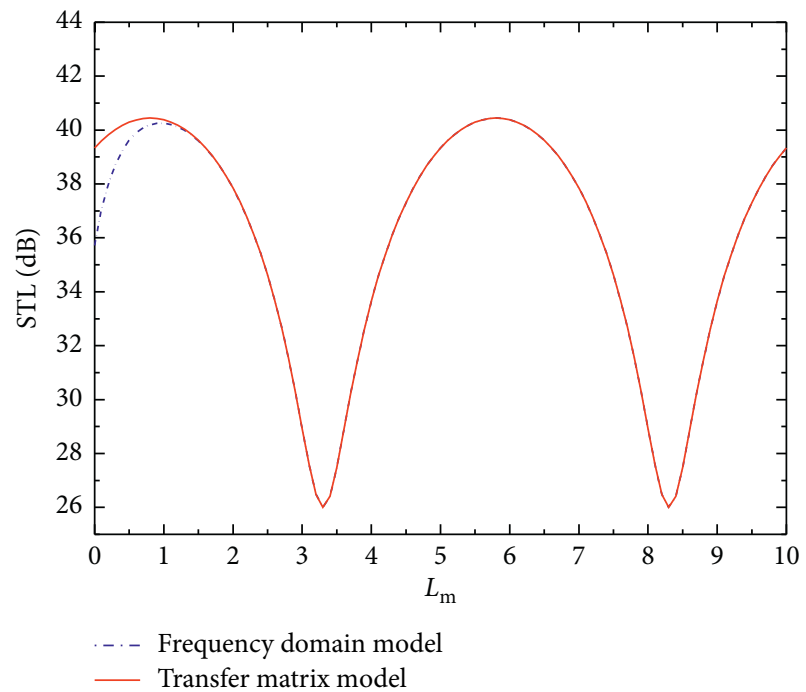

FIGURE 5: Influence of space between membranes on sound transmission loss. 


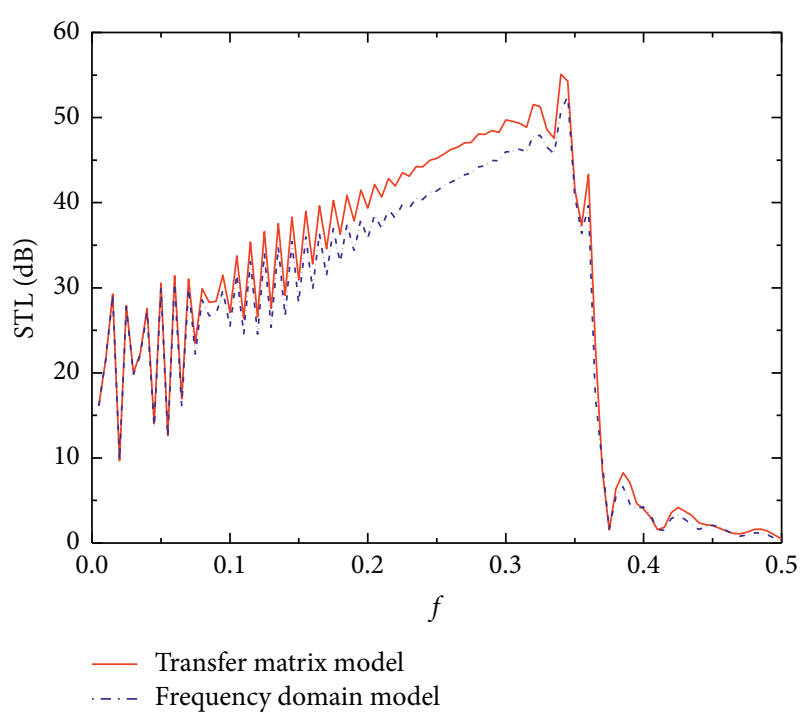

FIGURE 6: Variation of STL with incident sound frequency based on two different models.

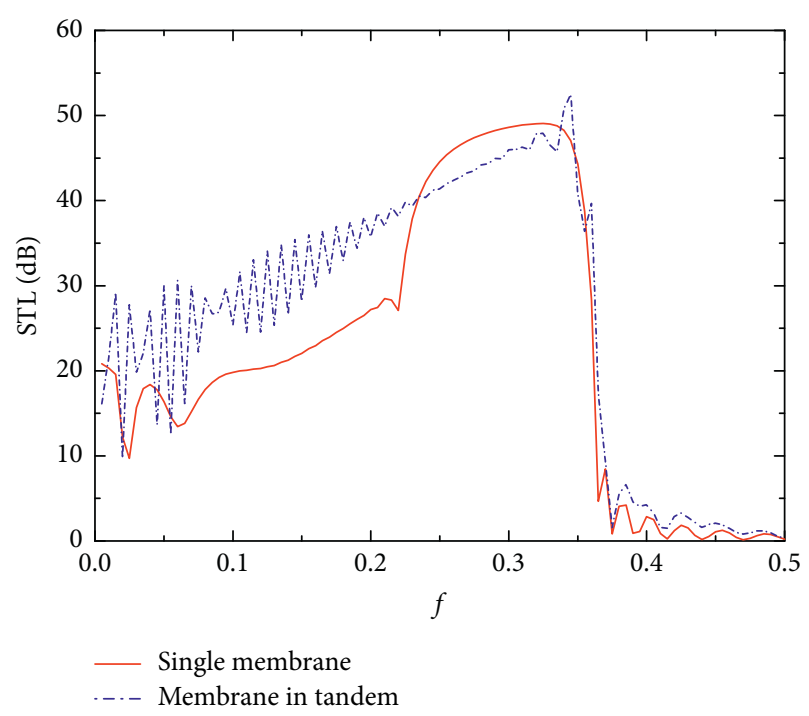

FIGURE 7: Comparison of STL between the single membrane setup and the membrane in tandem.

Furthermore, the variation of STL with incident sound frequency is shown in Figure 6 for gap $L_{m}=0$. It is found that there is no difference between these two models in low frequency range, which means coupling effect can be neglected in this situation. In the middle frequency range smaller than critical frequency, the discrepancy remains the same and $5 \mathrm{~dB}$ approximately. Notably, the quantitative deviation varies with specific structural and fluid parameters (i.e., heavy water case), and present numerical discussion highlights the coupling radiation effect owing to two membranes adequately.

4.4. Membrane in Tandem vs. Single Membrane. In contrast to the traditional one-membrane design $(L=20)$, the predictable advantage of multimembranes $\left(L_{1}=L_{m}=L_{2}=10\right)$ is larger transmission loss and lower requirement of tension force to maintain certain sound insulation effect if total length of membranes is fixed. As shown in Figure 7, STL of the membranes in tandem is higher than the single membrane case in the low frequency range (i.e., $f<0.24$ ). However, in the frequency range between $f=0.24$ and critical frequency region, the single membrane setup exhibits better sound insulation property than the membranes in tandem factually, which shows that it may be invalid to divide one complete membrane into two pieces for better sound insulation.

\section{Conclusions}

A completed coupling frequency domain model is developed to investigate the sound transmission loss property of the membrane in tandem. The validity and feasibility of the model is verified by comparing Huang's classical single membrane model and the authors' time domain model. The present model is capable of capturing the coupling effect between double membranes and helpful for further parameters' discussion and practical design.

\section{Data Availability}

The data used to support the findings of this study are included within the article.

\section{Conflicts of Interest}

The authors declare that they have no conflicts of interest.

\section{Acknowledgments}

This work was supported by the National Natural Science Foundation of China (11502110), Open Fund of the State Key Laboratory for Strength and Vibration of Mechanical Structures, Xi'an Jiaotong University (nos. SV2018-KF-01 and SV2018-KF-22), and Open Project of Space Structure and Mechanism Technology Laboratory of China Aerospace Science and Technology Group Co., Ltd. The third and fourth authors are grateful to the support from the Research Grants Council of the Government of Hong Kong Special Administrative Region, under Grant no. A-PolyU 503/15.

\section{References}

[1] B. Keshtegar, M. Bagheri, C. W. Fei, C. Lu, and D. K. Thai, "Multi-extremum-modified response basis model for nonlinear response prediction of dynamic turbine blisk," Engineering with Computers, 2021.

[2] L. Han, C. Chen, T. Guo et al., "Probability-based service safety life prediction approach of raw and treated turbine blades regarding combined cycle fatigue," Aerospace Science and Technology, vol. 110, Article ID 106513, 2021.

[3] C. W. Fei, H. T. Liu, S. L. Li, H. Li, L. Q. An, and C. Lu, "Dynamic parametric modeling-based model updating strategy of aeroengine casings," Chinese Journal of Aeronautics, 2021. 
[4] L. Huang, "A theoretical study of passive control of duct noise using panels of varying compliance," Journal of the Acoustical Society of America, vol. 109, no. 6, pp. 2805-2814, 2001.

[5] L. Huang, "Modal analysis of a drumlike silencer," Journal of the Acoustical Society of America, vol. 112, no. 5, pp. 20142025, 2002.

[6] L. Huang and Y. S. Choy, "Vibroacoustics of three-dimensional drum silencer," Journal of the Acoustical Society of America, vol. 118, no. 4, pp. 2313-2320, 2005.

[7] Y. S. Choy and L. Huang, "Experimental studies of a drumlike silencer," Journal of the Acoustical Society of America, vol. 112, no. 5, pp. 2026-2035, 2002.

[8] J. Du, Y. Liu, and Y. Zhang, "Influence of boundary restraint on sound attenuation performance of a duct-membrane silencer," Applied Acoustics, vol. 105, pp. 156-163, 2016.

[9] X. Yu, Z. Lu, L. Cheng, and F. Cui, "Vibroacoustic modeling of an acoustic resonator tuned by dielectric elastomer membrane with voltage control," Journal of Sound and Vibration, vol. 387, pp. 114-126, 2017.

[10] H. K. H. Fan, R. C. K. Leung, and G. C. Y. Lam, "Numerical analysis of aeroacoustic-structural interaction of a flexible panel in uniform duct flow," Journal of the Acoustical Society of America, vol. 137, no. 6, pp. 3115-3126, 2015.

[11] G. C. Y. Lam, R. C. K. Leung, H. K. H. Fan, and Y. Aurégan, "Effect of back cavity configuration on performance of elastic panel acoustic liner with grazing flow," Journal of Sound and Vibration, vol. 492, Article ID 115847, 2021.

[12] H. K. H. Fan, G. C. Y. Lam, and R. C. K. Leung, "Spatiotemporal aeroacoustic-structural responses of cavity-backed elastic panel liner exposed to grazing duct flow," Journal of Fluids and Structures, vol. 102, Article ID 103228, 2021.

[13] Y. S. Choy and L. Huang, "Multiple drumlike silencer for low frequency duct noise reflection," Applied Acoustics, vol. 70, no. 11-12, pp. 1422-1430, 2009. 